\title{
Clinical Batch
}

National Cancer Institute

\section{Source}

National Cancer Institute. Clinical Batch. NCI Thesaurus. Code C133992.

A batch produced for use in clinical trials. 